\title{
Challenges Encountered in Cancer Care and Management During Covid-19 in South Asian Countries
}

\author{
Sunishtha Singh Yadav ${ }^{1}$, Maryam Zain ${ }^{2}$, Pragati Sahai ${ }^{3}$, Shalini Porwal ${ }^{4}$, Vandana \\ Chauhan $^{1}$
}

${ }^{1}$ Centre for Medical Biotechnology, Amity Institute of Biotechnology, Amity University, Uttar Pradesh, India. ${ }^{2}$ Department of Biochemistry and Biotechnology, The Women University, Multan, Pakistan. ${ }^{3}$ Department of Biotechnology, School of Engineering \& Technology, Sharda University, Greater Noida, Uttar Pradesh, India. ${ }^{4}$ Amity Institute of Microbial Technology, Amity University, Uttar Pradesh, India.

\begin{abstract}
During the COVID-19, the cancer care is one of the most effected fields. The cancer care is either delayed or discontinued during the pandemic because of which cancer patients had to face resource constrain. Limited resources availability during this pandemic led to delay in the cancer diagnosis, undetected/ untreated cases and worse prognosis. The scenario of cancer care is even worse where the south Asian countries including India and Pakistan are considered due to unavailability of experts and adequate resources. Moreover, due to compromised immunity, the cancer patients are advised to restrict the number of visits to their Oncologist. Multipronged strategy to be included in different spheres of cancer treatment and cancer care. The ultimate motto of which is to ensure the well-being of cancer patients. The south Asian countries are framing different recommendations and guidelines to ensure the management of cancer patients through virtual/artificial intelligence modes, so that it shall promote contact less care and management of cancer patients in these countries. Since the beginning of Corona pandemic cancer care and research has been side lined all across the globe and in India \& Pakistan as well. This article discusses about effect of SARS-CoV-2 on cancer treatment and nursing in these countries. The strategies for cancer research have also been changed now to cancer care. Urgent need is there to find-out causes of delay in treatment and diagnosis on different stages of cancer. There is a need to minimise the obstacles if the pandemic continues over the coming months.
\end{abstract}

Keywords: Cancer care- South Asia- India- Pakistan- COVID-19

Asian Pac J Cancer Care, 5 (Suppl 1), 101-107

Submission Date: 06/27/2020Ａcceptance Date: 08/24/2020

\section{Introduction}

COVID-19 a pandemic that came as an infectious and contagious disease caused by recently found virus SARS-CoV-2 or Corona virus originated in Wuhan, China on December 2019 [1]. Breaking all the records in Wuhan, soon it spread to the entire world effecting drastically the most developed and medically advanced countries like Italy and United States [2]. The spread of virus from China soon turned into an outbreak causing deaths with respiratory and multiple organ failures worldwide especially seen in immunosuppressed individuals to an extent that WHO had to declare it as an emergency.

It declared and advisory stating that old people (above 60 years), kids (below 10 years), diabetic individuals, individuals with breathing troubles and Cancer patients should stay at home in quarantine to prevent themselves from COVID attack [3-4]. From studies conducted on several patients it was reported in Journal of Cancer Discovery in June 2020 that the patients with cancer are more prone to critical illness \& die than the infected general population [5]. According to the various cohort studies conducted in a hospital in Wuhan, China from the period of December 2019 till June 2020 it was

Corresponding Author:

Dr. Sunishtha Singh Yadav

Centre for Medical Biotechnology, Amity Institute of Biotechnology, Amity University, Uttar Pradesh, India.

Email: singhsunishtha@gmail.com 
analysed that overall cancer patients admitted during this period, 79\% were COVID positive in which $39 \%$ died in Intensive care unit while during the same period the general population of admitted to the same hospital was $37 \%$ COVID positive out of which $8 \%$ died, drawing the hypothesis that cancer patients are more vulnerable to the disease [6].

It was also observed through these cohort studies that the rate of fatality in case of COVID-19 was more due to multiple organ dysfunction than acute respiratory failure despite of the fact that it belongs to the family of SARS virus. This led to the explanation that this virus seriously affected the individuals with weak immunity like in Cancer patients that are undergoing chemotherapy and harmful medications. Such patients on attack with this virus developed multiple organ dysfunction more quickly than cancer patients who were not undergoing any chemotherapy [7].

Among the cancer patients it was found that the patients suffering from lung cancer, hematologic cancer and metastatic cancer stage IV were more prone to fatality due to COVID than nonmetastatic patients [8-9] and had more serious conditions. In addition to that the patients who had in past cancer surgery were also more prone to complexities of COVID to an extent of reversal of cancer or higher death rates [10].

The researchers and medical expert however do not conclude that COVID can cause fatality only to Cancer patients. There are several other factors that also came into light during these cohort studies. It was found that the patients suffering from cancer that died due to COVID were also older in age and some had history of smoking and breathing problems apart from going through chemotherapy [11]. One thing common all the fatal cases was poor or suppressed immune system in entire world including India.

\section{Relation of Immune system with COVID}

The ability of Virus to imitate the machinery of its host makes it difficult by our immune system or our defence system to locate any foreign particle especially if we have weak immune system. A healthy individual has strong force of defence cells inside the body that comprises of Major Histocompatibility Complex 1, Cytotoxic T cell, Helper T cells, Natural Killer cells, Macrophages etc. They all act as one big army that acts against any infection in our body. Virus usually release a protein called Viral interferons, that triggers the defence mechanism in the body. Once identified through MHC1 representation on infected cell, the cytotoxic cells release various enzymes to kill the virus by apoptosis. In case if Cytotoxic cells fail then macrophages and natural killer cells directly kill the infected cell with virus as a whole. So, it is seen that the machinery in our body is strong enough to combat any viral attack even if the virus mutates itself multiple times [12-13-14].

In cancer patients the tumour type, the stage of cancer, the age of patient, the type of therapy and supportive medications for cancer decide the rate of probability of contracting the viral infections. Such patients are immunocompromised because of antineoplastic therapy, chemotherapy (Steroid Supportive Medications) and immunosuppressive nature of cancer cells. Such cells alter the innate defence system of the body or may augment an untimely defence response like programme cell death/ apoptosis. Beside this usually the cancer patients that are old have other comorbidities and they have frequent visits to hospitals and nursing care, that further amplifies the complexities making them more prone to COVID attack [15]. So, it is clear from the above discussion that if one has strong immunity then chances of contracting COVID is less or if infected the severity of complication will be less and the recovery will be more.

\section{Management and treatment of Cancer in India during COVID-19}

India is a developing nation with upcoming medical facilities and updated medical care and to compare with medical giants like Italy, China, France and US is bizarre. Still India has put forward a strong front in combating the fatality of COVID-19. The ratio of fatality to the population of India effected is lower than the recovery ratio which was appreciated by WHO also [16]. The entire world is fighting the same catastrophe and experiencing the similar failures. For the first time entire world is united to fight the disaster with medical suggestions and practical approach taken by developed nation as the guiding light for health care workers and researchers worldwide [17]. But in India, the shoddy health care infrastructure, lack of good doctors and health care staff, inadequate knowledge and preparedness of pandemic and improper supply of resources has seriously compromised the patient care and safety of health workers. The hospitals being infected with COVID-19 has further worsen the situation. Even the Cancer Institute's scaled back after the reports received from China stated that COVID-19 is worse in Cancer patients [18]. With all these adversities and rationing in patient care the health workers in India designed their innate care delivery strategy especially for Cancer patients. The first strategy that was designed by two healthcare professionals of Tata Memorial hospital for cancer care delivery during COVID-19 as briefly mentioned below [19].

\section{COVID-19 measures at Tata Memorial Centre (19)}

\section{Management}

Formation of a committee known as COVID-19 task force $\&$ day by day briefings and formulating action plans.

\section{Cancer Care}

Avoiding complex medical procedures which requires various blood transfusions and delayed emergency care ward, Use of hypo-fractionated regimes at any point (eg, for breast, prostate, and lung cancers); arrangement of palliative radiotherapy in a uni-division/week by week course of action and Myelosuppressive systematic therapy should be decreased; moving on online specialists whenever possible; When extent of advantage is minimal the action should be postponed. 


\section{Directed- Patients}

Checking camps to be made outside the cancer centre which will lessen patient visits, limitation in family members \& companions in outpatient department and inpatient department, while routine follow ups should be changed to an online mode i.e. tele counsel.

4. Alerting Hospital

Foundation of set working systems for suspected cases/affirmed COVID-19 disease, body temperature facility and making of isolation area

5. Staff-Directed

Paid leave to be given to the individuals with high-risk (old aged staff, staff who is consuming immunosuppressive drugs, and pregnant staff); Shift changing of employees to guarantee an alternative in case of an occurrence of isolation and Provision to shift staff in emergency bus who are not able to arrive at work as a result of the transportation lockdown.

In India the hospitalisation is being given to most priority cases and mostly people if else healthy with no complications are advised self-isolation and quarantine for 14 days with basic medical and civic facilities from the state government. However, with cuts in hospitalisation and basic medical facilities the cancer patents are more prone to risk that otherwise is not life threatening at once. The cancer institutes and hospitals all over India are giving treatment first to the more curable cancer cases effected with COVID-19 that would benefit from the treatment over the non-curable ones that were as such in palliative care and the treatment would give only marginal effect. The decision helped in saving further the life of cancer patients with treatment for COVID-19 along with lifesaving cancer treatment. The decision seems harsh but in this pandemic time this is the only way to avoid regular cancer screenings and follow ups of cancer patients as to safeguard them from nosocomial infections related to infected hospitals and infected healthcare staff [20].

The establishment of National Cancer Grid that is the online web network of all the cancer centres in India was made by Tata Memorial Centre. This helped in sharing information and health protocols with the cancer specialist all over India through weekly webinars and webex. This is one of the most remarkable steps under Cancer Care strategy during COVID-19 by an institute. It has brought all the Cancer treatment and Preparedness for COVID-19 under one roof so that everywhere in India the cancer patients at this time are given similar lifesaving treatment [21].

The measures that are being taken by the health department of the State for safeguarding cancer patients is appreciable however, more important is preparedness of cancer patients by providing them complete knowledge of COVID-19 and its precautions. Informing them about basic hygiene, self-isolation, taking natural immunity boosters along with prescribed medicines and guiding them the new hospital care and visit only when it is very urgent [22]. To avoid increase of Coronavirus in Cancer patients the WHO guidelines i.e. isolate, test, treat and trace have to be adopted stringently for this Aarogya Setu app by Government is also very helpful. Through this app a Cancer patient can be aware of any active cases near his locality and the contaminant zones around his locality [23-24].

The Cancer centres through online follow ups with their patients are planning the treatment for them revolving around the strategies by Tata Memorial Centre [19], that has been broadly divided in categories: a) planned surgery- if required as per priority or can be delayed, b) undergoing immunotherapy, radiotherapy or chemotherapy- if necessary or can be delayed with specific life-saving cancer medications for time being, c) Stem cell transplantation- If not life threatening can be delayed and those with recent transplant should be kept in isolation to prevent them from COVID-19 infections, d) Antiviral therapy- this usually generated prophylactic response so yet it has to be confirmed that such therapy can be given to immunocompromised individuals, hence it should be avoided, e) antiviral medicines like Hydroxychloroquine, remdesivir7 etc are being given for treatment of COVID-19 but these drugs are not designed for COVID treatment so to try them on cancer patients is very risky and lastly (f) plasma therapy- The therapy involves transfusion of plasma cells carrying antibodies for COVID-19 from cured COVID-19 warrior to infected COVID-19 patient, thereby generating quick immune response in that patient and safeguarding him against this deadly virus [25]. Recently Delhi CM Arvind Kejriwal got approval for Plasma Therapy from ICMR to be conducted in hospitals named Rajeev Gandhi Super Speciality and LokNayak Jai Prakash for cancer patients after a successful clinical trial. It helped to moderate infected COVID-19 patients and is able to save their lives but in severe cases even this therapy is failing. So, Rajiv Gandhi Cancer Research Hospital is using this therapy on those cancer patients that are not very fatal and have chances to survive. These patients after therapy are given pulse oximeter to monitor their oxygen level daily [26].

So, it can be clearly stated that the effect of Cancer treatment during COVID-19 is shifted to Elective and Selective mode. The cancer centres as per WHO guidelines will isolate the patient first and if tested positive for COVID will be selected for life saving treatment and the surgery for the treatment of the cancer will be postponed by surgeon under elective surgery discipline that is depicted below [27]:

1. Examination of surgical need of cancer patient

2. Preparedness of Hospital with logistics and resource for the surgery

3. The consequences post-surgery to be mapped and aware to the patient

4. If delayed or postponed what risk it may impose to the patient

5. After analysis of above four points the final decision has to be taken.

This elective surgery discipline is very helpful in current scenario of cancer treatment in India as they are selected for cancer surgery only if they have two COVID-19 tests as negative after 72 hours. This step was taken because the cases of asymptomatic carriers is increasing in India in that case if inert COVID-19 
patient undergoes surgery it may lead to severe critical complications and immune suppressions in individuals leading to their death. So, it is advisable till it is not necessary all the surgeries should be deferred [28].

The nature fury or a bio weapon, the source of COVID-19 is still untraceable but the magnitude of its occurrence and prevalence has made it listed in one of the most-deadly disease in the world. It's completely delusional as to when the COVID-19 outbreak will be over so every state is doing it preparedness post COVID times as well. Healthcare professionals are being given psychological help and all the possible support and resource so that they remain to be the COVID -heroes. The researchers, DRDO, CSIR, ICMR and all the research labs in India are trying hard to develop a drug or vaccine to eliminate this virus. The Government on other hand has increased testing laboratories, makeshift isolation centres and hospitals while stocking the antiviral and antimalarial drug in store [29]. The population of India are utilising their lockdown period in developing immunity and hygiene in themselves and guiding others around them to do the same. Probably on brighter version of life Post COVID-19 the cases in Cancer should reduce as people are trying to be more fit by doing yoga and exercises. They are more hygienic and eating healthy food and maintaining balanced diet that builds their immunity. So, hope for a brighter future for India post COVID-19 [30].

\section{COVID-19 in Pakistan}

Corona virus started spreading in Pakistan in Late February, 2020. Initially the number of cases remained low as China borders closed and check on the International flights were performed. Later at the end of February it started rising as 3000 religious pilgrimage returned from Iran and Saudi Arabia, with many are the carriers. Although the Government officials were strongly criticised for chaotic response but even then, most of the people were quarantined in sub-optimal conditions. Some countries including China also helped the Pakistan Government for coping the condition. At the end of March an increase was seen as most of the Pakistanis overseas from heavily infected countries travel back [31-32]. Initially the complete lockdown started since 13th of March. The social distancing measures were advised, large gathering of religious congregation was not prescribed. The month of March and April remained closed for all educational institutes, offices and business. Nowadays, country is in a state of partial lockdown and they are reluctant for a complete lockdown because of fear of economic havoc as $25 \%$ of the population are on daily wages. New facts and data of India also suggest this type of fear is most likely to be found in the developing Asian countries [32]. Stabilizing the economic loss in front of the COVID-19 is an inevitable task and Government like Pakistan which falls under low and middle income is already facing it.

\section{Cancer care scenario in COVID era}

Pakistan, is very populous with a head count of 220 million people. With each passing year the rise in the new cases of cancer also increased each year with 200,000 each year. There are very few centres in this country. The main hospital that is dealing with cancer sufferers are Shaukatt Khannum Memorial Cancer Hospital and their centres for research. These hospitals are also overburdened with the patients. Every year 45,000 new patients come to register for treatment in these research hospitals. Due to the limitation in the capacity only 10,000 new cases are able to be accepted while the remaining cases are declined. Pakistan also has more than $11 \%$ of the patient's which falls under category of child and sufferers are not only of Pakistan origin but also from neighbouring countries as Afghanistan [31-32-33-34].

Although the cancer hospitals accept the patients free of charge irrespective of the race and nationality. But there is a lack of availability and resources to deal with such larger population of people. The national budget and health structure if fragile and patchy. With the advent of corona pandemic, the situation seems to become more worse as the country already has limited ventilators of 4000 working ventilators for a population of about 220 million.

Balancing the risk of COVID 19 with economic crisis, and starvation, and this task is unavailing for low- and middle-income countries people. Cancer care is a complex, expensive, time consuming and it is becoming difficult now for the patients and their families. The care of cancer is a complex procedure, it involves expensive and time consuming and is difficult even at the best of times. Economic uncertainty, as well as obligations on the normal and ongoing clinical check-ups because the pandemic complexes the complication of cancer [31-32-33-34].

Cancer care is a prolonged, and expensive treatment. It is difficult for the patients and their families to cope with it in normal circumstances. At present due to economic uncertainties and restrictions on the travel worldwide and within the country the care and treatment of this disease become more difficult. At the second largest city Lahore we have just 200 beds in Shaukat Khanum Memorial Cancer hospital, although it is considered a large hospital by public sector standards. Although for COVID care there are $1,000+$ bed hospitals in the city that have greater capacity to offer such care quarantine services. One of the major issues lacking in this country is ICU beds and ventilators. Shaukat Khanum Memorial Hospital devised a plan for increasing the number of ventilators from 11 to 15 by using available resources so that it reaches up to 50 ventilator beds. While normal inpatient beds have already converted to an ICU, to allow this expansion of beds [35-36].

The provincial Government is also supporting the cancer hospitals by giving the major equipment as primarily ventilators and cardiac monitors to monitor the cancer patients to operationalize the beds. Most of the hospitals are not taking the cancer patients most were not able to travel, in any case - and rapidly curtailed the visit of these patients for normal check-ups. The emergency cases including chemotherapy, radiation therapy is still continued. In addition to this a third inpatient unit has been opened for the patients having the coronavirus who are 
not in that stage to need an ICU, or have just recovered from ICU. The Shaukat Khanum hospital has recently made a decision that any spare beds are going to be used by the patients of corona patients who are unable to pay for their treatment [35-36].

Molecular pathology labs have started providing services for COVID-19 patients testing as part of effort against the coronavirus. Radiology services and essential imaging services are continued for COVID patients. While all elective services and screening procedures like endoscopy has been stopped. There is a nationwide shortage of the protective equipment's for COVID they include surgical gloves, N95 masks/ filtering face piece particle mask. Some of the developed textile industries has taken a step and started manufacturing gowns and other protective clothing, and several local manufacturers are now able to produce manufactured gowns and personal protective clothing (PPE). Efforts of producing N95 masks are also commenced but there is a concern to whether this can be equally efficient in current pandemic situation [31-32-33-34-35-36].

Pakistan National Disaster Management Authority has given the task to buy the personal protective clothing and then distribute to the needy people. The demand of PPE although has been increased globally and there is a global shortage and enormous demand everywhere in the world. In Pakistan there is a culture of attendants to accompany along with the patients. Normally three to four patients accompany to each patient. Now there is a restriction on the coming of attendants with the patients. There is also the initial screening available for all the people entering the hospitals by checking the common COVID-19 symptoms including fever, cough and breathlessness. All those patients are referred in separate building outside the main hospital. Following further treatment such patients are advised to self-isolate until their results are available. Moreover, the triage centre for patients are also opened 24 hours and number of patients seeing per day is increasing. The separate facility in hospitals like Shaukat Khanum and other cancer hospitals has helped the patients to be divided on the basis of treatment and now are identified easily. Separate routes of entering for chemotherapy patients are now available as to protect the chemotherapy patients or the patients having the severe cancer progression can be protected. This is now helping and protecting the cancer patients as till date the rise of the corona patients is increased in Pakistan with 1000 patients per day. There is a need in various hospitals to designate the parts as patients with COVID infection (red) suspicious (yellow) and non COVID (green) thus the easy identification and care could be established [35-36]. Most of the cancer and other hospitals has established the virtual clinics for identifying and separating patients and normal individuals. Pakistan is a country with very good mobile networks so almost $90 \%$ of the people have the mobile coverage. Pakistan has the highest rate of mobile penetration in South Asia so consultations are also being carried out by using the WhatsApp calling systems. Although many patients have not the WhatsApp calling but their response is positive with the relief that they are in touch with the medical specialists. Prescriptions and advices are also sent by using the SMS and screenshots services. The main patients facing the problem is about the purchase of medications of cancer as well. The people are in the financial crisis and supply of drug also changed. The strategies for treating the cancer patients having corona virus has also been changed. There is a need for revising the protocols for treating any patient of cancer affected by corona. Most of the liver cancer patients has been asked to take medications like oral sorafenib for the ones who do not need the immediate visits [31-32-33-34-35-36].

Those patients who are on chemotherapy have to wait for a longer period of time than the normal routine or they may be treated with the additional cycles to cope with long waiting time. In Pakistan since the beginning of COVID-19 in March 2020 new patients admitted for cancer treatment has been reduced to one third. The patients already having the radiation treatment has been reduced to half. According to one report out of normal treatment where 800 elective surgical procedures has been carried out since lockdown condition the rate of that procedures goes down to 20 [35-36].

There is also a need for counselling sessions for the healthcare and support staff who are working in this situation. Although some support session for cancer patients has already been setup by using the telemedicine facility. Most of the cancer clinics and hospitals has seen the rapid drop of the clinical income which are derived from the diagnostics services and pathological collection. Some of the hospitals have also cut down the salary of the staff by $10 \%$. Ranging from $25 \%$ reduction of highest paid and $5 \%$ reduction of lowest paid workers. New doctors and cancer professionals are also now hard to train and retained in resource limited environment. The patients with some associated reasons are only admitted based on variety of factors as age, availability of appropriate drugs, likelihood of having the complete cure etc. While most hospitals have already developed the system to accept the patients based on the severity of disease. To date Pakistan has $199 \mathrm{~K}$ confirmed cases with 4000 deaths and 2729 critical patients. The cancer treatment and check-ups are now reduced which will affect the survival and prognosis of cancer patients. There is a need to keep going the normal check-up and facility availability to cancer patients so that they may not suffer from corona pandemic. Immediate strategies for care for cancer and corona effected patients need to be devised [31-32-33-34-35-36].

\section{Challenges in the minds of people: Questions raised in people's mind due to fear}

Patients have developed fear, a very dark fear due to COVID-19. If left unanswered these may cause anxiety in public and turn the situation in the worst. Therefore, it is important to address these questions.

"Is cancer or COVID-19 going to execute them?"

"What might be the smart solution for patients to do?

"Consulting doctors are saying they have to hold and sit tight for the treatment?"

"How might they save themselves?"

There is a big question in the minds of patients "What 
if cancer increases, will all the past treatment they had will be effective?" "No one is able to tell anything even doctors are scared to treat cancer patients. This obviously portray stress and a decrease in patients to the hospitals.

In conclusion, care for cancer patients has been majorly hit all over the world. In India and Pakistan as well, cancer care is affected badly. The serious issues in these times of COVID is faced by Cancer patients because of their weak immune system as they are more prone to this Corona Virus disease which the world is facing. Mortality of cancer patients has increased as cancer treatment has been stopped and many hospitals has been totally converted to COVID Hospitals for treatment of Corona. Travel limitations was also imposed by the central and the state government of India which also reduced access to the hospitals during the time of lockdown. Many of the hospitals has also stopped it's OPD and delaying/cancelling hospital visit of cancer patients to protect them from corona disease. Many questions have been raised and is a big challenge for all the hospitals and doctors such as Would it be right to proceed or begin chemotherapy/radiotherapy/directed treatment/hormonal treatment during these COVID times? What kinds of medical surgery shall be possible during this pandemic in cancer patients? What shall be the ideal time for medical procedure? There is an urgent need to expedite and formulate new methods and techniques to treat, give timely advice and care for the cancer patients. As India \& Pakistan are now at peak of corona pandemic, strategies that prove effective against the corona virus need to be devised. There is also a need to gather cancer professionals for implications of treatment strategies for seriously ill patients. Government has to plan the exit strategy for the cancer patients who cannot wait for the treatment.

\section{References}

1. Guan W, Ni Z, Hu Y, Liang W, Ou C, He J, Liu L, Shan H, Lei C, Hui DS, Du B, Li L, Zeng G, Yuen K, Chen R, Tang C, Wang T, Chen P, Xiang J, Li S, Wang J, Liang Z, Peng Y, Wei L, Liu Y, Hu Y, Peng P, Wang J, Liu J, Chen Z, Li G, Zheng Z, Qiu S, Luo J, Ye C, Zhu S, Zhong N. Clinical Characteristics of Coronavirus Disease 2019 in China. New England Journal of Medicine. 202004 30;382(18):17081720. https://doi.org/10.1056/nejmoa2002032

2. Wu Z, McGoogan JM. Characteristics of and Important Lessons From the Coronavirus Disease 2019 (COVID-19) Outbreak in China. JAMA. 202004 07;323(13):1239. https:// doi.org/10.1001/jama.2020.2648

3. World Health Organization (WHO) (2020, February 11). Key considerations for repatriation and quarantine of travellers in relation to outbreak of novel coronavirus2019-nCoV. Retrieved from https://www.who.int/ith/Repatriation Quarantine_nCoV-key-considerations_HQ-final11Feb..

4. Liang W, Guan W, Chen R, Wang W, Li J, Xu K, Li C, Ai Q, Lu W, Liang H, Li S, He J. Cancer patients in SARSCoV-2 infection: a nationwide analysis in China. The Lancet Oncology. 2020 03;21(3):335-337. https://doi.org/10.1016/ s1470-2045(20)30096-6

5. Dai M, Liu D, Liu M, Zhou F, Li G, Chen Z, Zhang Z, You H, Wu M, Zheng Q, Xiong Y, Xiong H, Wang C, Chen C, Xiong F, Zhang Y, Peng Y, Ge S, Zhen B, Yu T, Wang L, Wang H,
Liu Y, Chen Y, Mei J, Gao X, Li Z, Gan L, He C, Li Z, Shi Y, Qi Y, Yang J, Tenen DG, Chai L, Mucci LA, Santillana $\mathrm{M}$, Cai H. Patients with cancer appear more vulnerable to SARS-COV-2: a multi-center study during the COVID-19 outbreak. Cancer Discovery. 202004 28;:CD-20-0422. https://doi.org/10.1158/2159-8290.cd-20-0422

6. Zhang L, Zhu F, Xie L, Wang C, Wang J, Chen R, Jia P, Guan H, Peng L, Chen Y, Peng P, Zhang P, Chu Q, Shen Q, Wang Y, Xu S, Zhao J, Zhou M. Clinical characteristics of COVID-19-infected cancer patients: a retrospective case study in three hospitals within Wuhan, China. Annals of Oncology. 2020 07;31(7):894-901. https://doi.org/10.1016/j. annonc.2020.03.296

7. Kuderer NM, Choueiri TK, Shah DP, Shyr Y, Rubinstein SM, et al. Clinical impact of COVID-19 on patients with cancer (CCC19): a cohort study. The Lancet. 2020 06;395(10241):1907-1918. https://doi.org/10.1016/s01406736(20)31187-9

8. Blimark C, Holmberg E, Mellqvist U, Landgren O, Bjorkholm M, Hultcrantz M, Kjellander C, Turesson I, Kristinsson SY. Multiple myeloma and infections: a population-based study on 9253 multiple myeloma patients. Haematologica. 2014 Oct 24;100(1):107-113. https://doi.org/10.3324/ haematol.2014.107714

9. Covid, C. D. C., and Response Team. "Severe outcomes among patients with coronavirus disease 2019 (COVID-19)United States, February 12-March 16, 2020”. MMWR Morb Mortal Wkly Rep . 2020;69(12):343-346.

10. Wang H, Zhang L. Risk of COVID-19 for patients with cancer. The Lancet Oncology. 2020 04;21(4):e181. https:// doi.org/10.1016/s1470-2045(20)30149-2

11. Zhang, Bicheng, Xiaoyang Zhou, Yanru Qiu, Fan Feng, Jia Feng, Yifan Jia, Hengcheng Zhu, et al. "Clinical characteristics of 82 death cases with COVID-19". MedRxiv (2020).

12. Roitt, Ivan Maurice. Essential immunology. No. 2nd edition. 1974.

13. Kindt, Thomas J, Richard A, Goldsby, Barbara A, Osborne, Janis Kuby. Kuby immunology. Macmillan, 2007.

14. Finn OJ. Cancer Immunology. New England Journal of Medicine. 200806 19;358(25):2704-2715. https://doi. org/10.1056/nejmra072739

15. Warner, Jeremy Lyle, Sam Rubinstein, Petros Grivas, Toni K. Choueiri, Nicole Maria Kuderer, Dimpy Shah, Donna R. Rivera et al. "Clinical impact of COVID-19 on patients with cancer: Data from the COVID-19 and Cancer Consortium (CCC19)." (2020): LBA110-LBA110.

16. https://www.who.int/india/emergencies/coronavirus-disease(covid-19).

17. Al-Shamsi HO, Alhazzani W, Alhuraiji A, Coomes EA, Chemaly RF, Almuhanna M, Wolff RA, Ibrahim NK, Chua ML, Hotte SJ, Meyers BM, Elfiki T, Curigliano G, Eng C, Grothey A, Xie C. A Practical Approach to the Management of Cancer Patients During the Novel Coronavirus Disease 2019 ( COVID -19) Pandemic: An International Collaborative Group. The Oncologist. 202004 27;25(6). https://doi.org/10.1634/theoncologist.2020-0213

18. Asokan I, Rabadia SV, Yang EH. The COVID-19 Pandemic and its Impact on the Cardio-Oncology Population. Current Oncology Reports. 202005 28;22(6). https://doi. org/10.1007/s11912-020-00945-4

19. Pramesh C, Badwe RA. Cancer Management in India during Covid-19. New England Journal of Medicine. 2020 05 14;382(20):e61. https://doi.org/10.1056/nejmc2011595

20. Sharma D, Agrawal V, Agarwal P. Roadmap for Restarting Elective Surgery During/After COVID-19 Pandemic. Indian 
Journal of Surgery. 2020 06;82(3):235-239. https://doi. org/10.1007/s12262-020-02450-1

21. https://tmc.gov.in/ncg/.

22. Gupta, Nivedita, Ira Praharaj, Tarun Bhatnagar, Jeromie Wesley Vivian Thangaraj, Sidhartha Giri, Himanshu Chauhan, Sanket Kulkarni, et al. "Severe acute respiratory illness surveillance for coronavirus disease 2019, India, 2020". Indian Journal of Medical Research 151. 2020;2:236.

23. World Health Organization (WHO) (2020, March 18). WHO Director-General's opening remarks at the media briefing on COVID-19. Retrieved from https://www.who.int/dg/ speeches/detail/who-director-general-s-opening-remarksatthe-media-briefing-on-covid-19---18-march-2020.

24. Jhunjhunwala, Ashok. "Role of Telecom Network to Manage COVID-19 in India: Aarogya Setu.". Transactions of the Indian National Academy of Engineering. 2020;:1 .

25. https://www.thehindu.com/sci-tech/science/plasma-therapyfor-covid-19-patients/article31373992.ece.

26. https://www.hindustantimes.com/delhi-news/plasmatherapy-helping-moderate-covid-19-patients-stabilise-delhicm-kejriwal/story-dlj2zUzL2bW6OHMyMZaxRK.html..

27. Spinelli A, Pellino G. COVID-19 pandemic: perspectives on an unfolding crisis. British Journal of Surgery. 202003 23;107(7):785-787. https://doi.org/10.1002/bjs.11627

28. Nahshon C, Bitterman A, Haddad R, Hazzan D, Lavie O. Hazardous Postoperative Outcomes of Unexpected COVID-19 Infected Patients: A Call for Global Consideration of Sampling all Asymptomatic Patients Before Surgical Treatment. World Journal of Surgery. 202005 16;44(8):24772481. https://doi.org/10.1007/s00268-020-05575-2

29. Indian Council of Medical Research (ICMR). (2020, March 20). Revised Strategy of COVID19 testing in India. https://www. mohfw.gov.in/pdf/ICMRrevisedtestingstrategyforCOVID. pdf.

30. Sharma, Dhananjaya, Vikesh Agrawal, Pawan Agarwal. "Roadmap for Restarting Elective Surgery During/After COVID-19 Pandemic". Indian Journal of Surgery. 2020;:1-5.

31. International Agency for Research on Cancer Globocan (2018) Pakistan fact sheet [https://gco.iarc.fr/today/data/ factsheets/populations/586-pakistan-fact-sheets.pdf].

32. Yusuf MA, Hussain F, Sultan F, Badar F, Sullivan R. Cancer care in times of conflict: cross border care in Pakistan of patients from Afghanistan. ecancermedicalscience. 202003 05;14. https://doi.org/10.3332/ecancer.2020.1018

33. Bhutta ZA, Basnyat B, Saha S, Laxminarayan R. Covid-19 risks and response in South Asia. BMJ. 202003 25;:m1190. https://doi.org/10.1136/bmj.m1190

34. India's jobless rate swells above $23 \%$ amid lockdown, survey shows (2020) Times of India [https://timesofindia. indiatimes.com/business/india-business/indias-joblessrate-swells-above-23-amid-lockdown-survey-shows/ articleshow/75026539.cms].

35. Government COVID data. http://covid.gov.pk/.

36. Yusuf A. Cancer care in the time of COVID-19-a perspective from Pakistan. ecancermedicalscience. 202004 20;14. https://doi.org/10.3332/ecancer.2020.1026

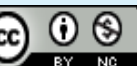

This work is licensed under a Creative Commons AttributionNon Commercial 4.0 International License. 